\title{
A Literature Survey on Flipped Class Room
}

\author{
Manoj Kumar P. \\ Department of Computer Science, \\ CUCEK,CUSAT.
}

\begin{abstract}
The flipped class room employs asynchronous video lectures and practice problems as homework and the valuable class time for in-class activities is spend with active learning techniques and one-on-one time with students. In this survey the effectiveness of flipped classrooms in a wide range of subjects like Engineering, Education Technology, Mathematics and Statistics, Linear Algebra, English language course,Calculus, Psychology Course, Nursing Education, Higher Education Environmentand Computer programming is dealt with. The study found that flipped class rooms were more effective compared to traditional class rooms in all the above courses.
\end{abstract}

Keywords-Flipped Class Room, Traditional class Room, Active Learning,Survey.

\section{INTRODUCTION}

There have been several studies on the effectiveness of flipped classrooms in a wide range of subjects like Engineering, Education Technology, Mathematics and Statistics, Linear Algebra, English language course, Calculus, Psychology Course, Nursing Education, Higher Education Environment, Faculty Training Programmeand Computer programming. Engineering, Social Science, Education Technology, Mathematics and Statistics, Linear Algebra, English language course,Calculus, Psychology Course, Nursing Education, Higher Education Environment and Computer programming. Very less works was done in this area which checks the effectiveness of flipped class room in these above domains. Here the author studies the flipped class room strategy employed in each area and reports the pros and cons found in the above areas. The study found that flipped class rooms were more effective compared to traditional class rooms in all the above areas. In future more courses will switch to flipped class rooms because of the availability of internet at a cheaper rate. Since the flipped class rooms employ active learning techniques it helped to develop exploratory and inventive skills in students, improved students' innovative consciousness and attitudes etc.

\section{Literature Survey}

Bishop and Verleger has conducted a comprehensive survey on flipped classrooms. Studies showed that flipped class rooms aroused a mixed response among the students. The overall response among the students was however positive. Students preferred teachers to take class rather than watching video lectures but prefer interactive class room activities over lectures. Evidences revealed that students learning has considerable improved for the flipped version compared to the traditional one. However, one of the major pitfalls of flipped learning is that very little work is carried out investigating learning outcomes objectively[1].
Stephanie Gray Wilson redesigned a statistical course to a flipped class room in order to increase student interest, engagement and retention of the types of statistical knowledge and skills needed for students to be successful in their academic and professional careers. Quizzes were introduced regularly in the flipped classrooms in order for the students to read the text book material.48.2\% of the students indicated that it was "somewhat helpful" or "very helpful". $58 \%$ of the students agreed that reading the text book was either somewhat helpful" or "very helpful" in obtaining the learning objectives of the course. In addition to the text books ,online resources form Khan Academy(www.khanacademy.org) were provided.41\% of the students indicated that they found the site either "somewhat helpful" or "very helpful" in obtaining the learning objectives of the course. A learning group was created among the students for forming a support network within the class. This increased interaction among students before and after the class. Group home works were also provided so that students work with their learning groups to check answers during class on the day of the assignment is due. During discussion of homework, the instructor was available to guide the students on their answers. Even though there was overall improvement in students' grade, some students responded that the lack of lecture as a problem because they have to teach themselves an entire chapter of statistics. However, the changes imparted created a positive impact on students' attitude towards the class and it also reported a huge improvement in students' performance[11]. Diane Billings flipped lectures and discussions in Nursing Education by promoting more meaningful interactions between learners and educators. In a flipped class room the task of memorizing facts is replaced with tasks applying in a real-world clinical situation. Students work individually or in groups to solve clinical cases and develop care plans, which results in individualized learning, ample feedback and ongoing assessment of learning outcomes. In a flipped class room learners can choose from a variety of pre-class learning resources such as prerecorded lectures and reading assignments on the basis of their learning style. Using the resources before the class help the students to read or listen to the course material several times before applying it in the class room. For some students the new role of active and engaged learner may be challenging and they may resist the need to spend more time preparing for class. So, the educator who used a flipped class room must convince the learners the purpose, benefits and expected outcomes of the flipped class room. Evidence have shown that flipping the class room is an effective strategy for helping learners to better connect learned concepts to clinical practice and for preparing them for clinical experiences. Research shows that there is a considerable improvement in exam scores for 
students who employs flipped class room. But students' satisfaction was less because flipped class room requires more work compared with the traditional approach [12].

Herala et al. introduced programming courses in a flipped method of learning. The results showed that flipped class room model found to be more efficient than the traditional class room. Since the students are familiar with the basics before arriving to the class collaborative methods like peer learning and peer teaching where students are active audience in teaching took place. The learning activities outside class room are done with reading programming literature and watching videos and the class is controlled with quizzes that present questions in the weekly changing topic. The major advantage of flipped class room is that students can learn at their own pace and teachers can concentrate on actual problems instead of repeating the basics in the class room and no costly lecturing is needed. At the end it has shown that students learning has improved [2]. Hsieu-Ting Hung examined the effects of flipping the class room for English language learning course. The results showed that flipped class room showed significant improvement in student performance compared to the traditional one. The results also showed that there is a positive effect on students learning attitudes. The flipped model identified four major pillars of FLIP, namely, flexible environment, learning culture, intentional content and professional educator. The first pillar suggests that students can decide where, when and how they view the material. In language learning the flexibility of accessing the focal material or linguistic input anywhere any time and at own paces is a practical approach to lowering the anxiety and frustration of learners. The second pillar suggested a paradigm shift in responsibility from teachers to learners resulting in a student-centered learning culture. Another important consideration in flipped class rooms is the use of intentional content to foster student learning in pre-class and in class activities, as represented by the third pillar of the flipped class room learning model. The fourth pillar specifies the teachers' skill in controlling the classes. The teacher is empowered with knowledge, skills and autonomy. Teacher can reconstruct their way of thinking and teaching in response to the changing demands of the teaching profession[3].

Gregory S. Masol et al. conducted inverted class room in a control systems course in the department of Mechanical Engineering at Seattle University, USA. The two-year course was designed in such a way that the first year was taught using traditional class room where as the second year was taught using inverted class room. The study revealed that the content coverage in the inverted class room were much more compared to the traditional class room. Students participating in the inverted class room performed as well or better on comparable quiz and exam questions and on open ended design problems. Since the inverted class room was new to the students they struggled initially with the new format but adapted quickly and found the inverted class room format to be satisfactory and effective [4].

Mikael Cronhjort et al. replaced traditional lecture-based teaching in calculus with a flipped class room approach. The study found that the failure rate was less and highest grade increased more in the flipped class room compared to the lecture group, indicating that the intervention was beneficial for low as well as high performing students. A calculus base line test was conducted which indicated a higher gain in the flipped class room compared to the lecture group. The engagement survey showed that students felt more engaged in the flipped group. In the flipped class rooms due to active learning techniques students felt that teachers care more about their learning, take their questions more seriously and they received more support. The social aspects of peer discussions in the teacher-based sessions of flipped class rooms also seemed to improve student learning [5].

Heather D Hussey et al. flipped a psychology course. Flipping the class room is a solution for problems like lack of student engagement during class, passive learning through lectures, and ineffective lecturers. Research shows that students engaged in the flipped course design enjoy or prefer the course structure, regardless of actual learning outcomes. Students in the flipped course academically outperformed students in the traditional course. Learning takes place more in the flipped course compared with the traditional course. Faculties who employed flipped class rooms found that they are more enjoyable to teach, student involvement was high, one to one interaction with the students was also high and found greater enjoyment in the interactions [6].

Mike Mavromihales and Violeta Holmes flipped a module of the manufacturing technology and practical workshop-based work course in engineering and technology studies. Power point presentations, detailed notes, audio visual materials including YouTube videos were provided to watch and study as part of pre-class activity and the in-class activity are devoted to exercises, projects and discussion. The objective of the study was to examine whether the flipped class room approach can enhance the learning experience of the students through better engagement with the students compared with the traditional class rooms. Feedback questionnaires was given to hundreds of students and the level of student participation and level of success has been prepared by peer observation. The observations showed that there was significant improvement in learning experience and the results of the course outcomes has improved [7].

ManojKumar.P et al. taught programming in java to the under achievers using the flipped approach and the high achievers using traditional approach. They examined how the underachievers performed in comparison with the high achievers after undergoing flipped class room sessions. At the end of the sessions the results showed that low achievers performed at par with the high achievers of the traditional class room. Traditional class rooms consists of lecturing as the in-class activity and home work as the out-class activity whereas the flipped class rooms were equipped with watching videos as the outclass activity and the major inclass activity was think-pair-share. In the light of this study it can be concluded that flipped class rooms are a good method to teach underachievers, specifically for courses like programming which require more scaffolding [8].

B.Schmidt introduced flipped class room in a first semester engineering dynamics course. Here the video lectures which are the pre-class activities are replaced with pen casts. Pen casts are used to record handwritten notes in real time along 
with the instructor's vocal explanation. At the lectures the students were asked to vote by using clickers and the results of the voting were used to stimulate peer discussion.After the peer discussion the students were asked to vote again on the same questions. The second votes results were much improved because it was after the peer discussions. A student or a teacher explained the solution to the students. The remaining part of the lecture was given in a traditional format with lots of text book exercises to work as homework and the answers to these exercises were uploaded in the course home page. One class was taught by using traditional method while the other class was taught by the method described above by the same teacher. The results showed that the learning outcome for flipped class room was high for the engineering dynamics course [9].

S.se et al. introduced flipped class room in a Linear Algebra course. Pre class video lectures were sent to the students and the students were asked to send the points he/she is unable to pick up before the class. This helped the teacher to focus on the weaker sections of the class and enable the teacher to bring out best possible solutions to clarify the concepts. New computational experiments were designed in such a way as to engage participation of learners in the class room. As a result of flipped class rooms active learning took place and the learning was more effective [10].

\section{CONCLUSION}

In a traditional class room learners can easily forget what is taught 15 minutes back and there is no way to rewind it for rehearing. In flipped class roomsthe pre-class video lectures are eternal in nature they can be viewed at any number of times at their own pace and time. In this survey the effectiveness of flipped class rooms in a variety of subjects like Engineering, Education Technology, Mathematics and Statistics, Linear Algebra, English language course,Calculus, Psychology Course, Nursing Education, Higher Education Environment and Computer programming is dealt with. The study analyzed the outcome of employing flipped class rooms in each of these above courses. In all the above course employing flipped class rooms was found to be effective and the students performed satisfactorily.

\section{REFERENCES}

[1] J.L.Bishopand M.A. Verleger, "The flipped classroom: a survey of the research," Proceedings of the $120^{\text {th }}$ ASEE Annual Conference and Exposition, 2013.

[2] A.Herala, A.Vanhala, A. Knutas.andJ. Lkonen,"Teaching programming with flipped classroom method: a study from two programming courses," Proceedings of the $15^{\text {th }}$ Koli Calling Conference on Computing Education Research, 165-166, 2015.

[3] H.Hung, "Design-based research: redesign of an english language course using a flipped classroom approach,'TESOL International Association,51(1), 2017.

[4] G.S.Mason, T. Shuman, and K.E. Cook, "Comparing the effectiveness of an inverted classroom to a traditional classroom in an upperdivision engineering course," IEEE Transactions on Education,56(4),430-435,2013.

[5] Mikael Cronhjort, Lars Filipsson, Maria Weurlander, "Improved engagement and learning in flipped-classroom calculus," Teaching Mathematics and its Applications: An International Journal of the IMA,37(3),2018.
[6] Heather D Hussey, Aaron S. Richmond, Bethany Fleck, "A Primer for Creating a Flipped Psychology Course," Psychology Learning and Teaching,14(2),2015.

[7] Mike Mavromihales and Violeta Holmes, "Delivering manufacturing technology and workshop appreciation to engineering undergraduates using the flipped classroom approach," International Journal of Mechanical Engineering, 44(2), 2016.

[8] ManojKumar.P, Renumol V.G and Sahana Murthy, "Flipped Class room Strategy to Help Underachievers in Java Programming," International Conference on Learning and Teaching in Computing and Engineering , 1,44-49, 2018

[9] B.Schmidt, "Improving motivation and learning outcome in a flipped classroom environment," IEEE International Conference on Interactive Collaborative Learning, 2014.

[10] S.Se,B.Ashwini, and A.Chandran, "Computational thinking leads to computational learning: flipped classroom experiments in linear algebra,"IEEE International Conference on Innovations in Information, Embedded and Communication Systems, 2015.

[11] Stephanie Gray Wilson, "The Flipped Class: A Method to Address the Challenges of an Undergraduate Statistics Course," Teaching of Psychology, 40(3), 2013

[12] Diane Billings, "'Flipping' the Class room," American Journal of Nursing, 116(9), 2016 